\title{
Leiomyosarcoma of the splenic vein with a history of bilateral retinoblastoma: A case report and review of the literature
}

\author{
MIHO AKABANE ${ }^{1}$, MASAJI HASHIMOTO ${ }^{1}$, YUTAKA TAKAZAWA ${ }^{2}$, \\ DAISUKE HATTORI ${ }^{3}$, RIKAKO KOYAMA ${ }^{3}$ and TSUNAO IMAMURA ${ }^{3}$ \\ ${ }^{1}$ Division of Hepatobiliary-Pancreatic Surgery, Department of Gastroenterological Surgery, \\ Departments of ${ }^{2}$ Pathology and ${ }^{3}$ Gastroenterology, Toranomon Hospital, Tokyo 105-8470, Japan
}

Received August 29, 2021; Accepted November 9, 2021

DOI: $10.3892 / \mathrm{mco} .2021 .2468$

\begin{abstract}
Diagnosing leiomyosarcomas of the splenic vein is challenging, and a treatment strategy has not yet been established for this condition. We herein report the case of a 45-year-old female patient with a history of retinoblastoma who underwent pancreatosplenectomy for a primary leiomyosarcoma originating from the splenic vein and transcatheter arterial chemoembolization for metastatic hepatic lesions observed 5 months postoperatively. An initial medical check-up using abdominal ultrasound revealed a $40-\mathrm{mm}$ mass behind the pancreatic tail. Imaging tests revealed a well-circumscribed mass compressing the pancreas posteriorly, without invasion into the pancreatic duct. The splenic vein was torn, with the epiploic veins developed as collateral blood vessels, which was an atypical finding for carcinoma. The patient was followed up regularly. At 5 years after the first visit, an abdominal ultrasound showed that the mass had increased in size to $50 \mathrm{~mm}$, and had developed into a tumor embolus within the splenic vein, causing an obstruction of the vessel. Pancreatosplenectomy was performed due to suspicion of malignancy. The final diagnosis was leiomyosarcoma arising from the splenic vein. The patient was discharged on postoperative day 15 and was followed up regularly thereafter. Imaging studies performed 5 months postoperatively revealed four hepatic lesions. The hepatic masses were histologically diagnosed as metastatic leiomyosarcomas, and transcatheter arterial chemoembolization was performed using epirubicin. Since then, regular follow-ups have been conducted without observed recurrence. Leiomyosarcoma of the splenic vein is exceedingly rare, and the number of reported cases is not sufficient to establish clinical guidelines. Therefore, it is crucial to
\end{abstract}

Correspondence to: Dr Miho Akabane, Division of HepatobiliaryPancreatic Surgery, Department of Gastroenterological Surgery, Toranomon Hospital, 2-2-2 Toranomon, Minato, Tokyo 105-8470, Japan

E-mail: akabane.miho@gmail.com

Key words: leiomyosarcoma, splenic vein, surgery, transcatheter arterial chemoembolization, retinoblastoma collect more reports on the occurrence and treatment of this disease.

\section{Introduction}

Primary leiomyosarcoma usually occurs in the fifth and sixth decades of life. The most common clinical symptoms are abdominal pain and gastrointestinal bleeding (1). This type of tumor originates from smooth muscle cells and mainly occurs in the retroperitoneum and abdomen (2). The overall incidence of vascular leiomyosarcoma has been reported to be $<2 \%$ (3). Complete surgical resection is considered to be the cornerstone of treatment for localized vascular leiomyosarcomas, while the role of neoadjuvant chemotherapy and radiation remains unknown (4). The prognosis of patients with vascular leiomyosarcoma is reportedly poorer compared with the prognosis of patients with leiomyosarcomas of different origins (4). Primary leiomyosarcoma arising from the splenic vein is an extremely rare occurrence and its clinical features are incompletely understood. The clinical diagnosis of this condition remains challenging, despite advancements in imaging modalities, owing to its rarity. Furthermore, evidence regarding treatment strategies, including postoperative adjuvant radiation and chemotherapy, is scarce. We herein describe the case of a patient with a history of retinoblastoma who was diagnosed with leiomyosarcoma originating from the splenic vein and was treated with pancreatosplenectomy and subsequent transcatheter arterial chemoembolization due to the development of hepatic metastases 5 months postoperatively (5). The aim of the present report was to help with the development of clinical guidelines for leiomyosarcomas of the splenic vein.

\section{Case report}

A 45-year-old Japanese woman, who had a history of infantile retinoblastoma treated by enucleation and chronic hepatitis $\mathrm{C}$ diagnosed in her twenties, underwent a routine medical check-up at Hiroo Medical Clinic (Tokyo, Japan). In May 2015, the patient presented to the hospital with no apparent symptoms. Abdominal ultrasonography revealed a mass $\sim 40 \mathrm{~mm}$ in size located posterior to the pancreatic tail. The pancreatic body was observed to be distended dorsally on CT examination. The adrenal gland was independently identified, and 
it was determined that the lesion did not originate from the adrenal gland. In addition, the lesion appeared to have no border with the pancreatic parenchyma and had the same signal intensity as the pancreas. There was a tear in the splenic vein, and the epiploic veins were highly developed as collateral blood vessels, which is an atypical finding in carcinoma. On MRI examination, the lesion appeared as a well-circumscribed mass that compressed the pancreas posteriorly with no invasion into the pancreatic parenchyma. The signal intensity of the mass was lower on T1-weighted images and higher on T2-weighted images as compared to that of the pancreas. The signal intensity of the lesion on diffusion-weighted images was high and that on an apparent diffusion coefficient map was low, suggesting that the lesion had a high cellular density. The value of the apparent diffusion coefficient was 0.911 . Although the pattern of these signal intensities was similar to that of the spleen, the signal intensity of the lesion on T2-weighted images was lower than that of the spleen (Fig. 1). Ferucarbotran-enhanced MRI performed 1 month after the patient's initial visit revealed that the lesion had a higher signal intensity than the spleen, indicating that the lesion was not an accessory spleen. Furthermore, the lesion had not increased in size in 1 month, indicating that it was possibly not a malignant tumor. Therefore, the lesion was suspected to be an extrapancreatic tumor, such as a retroperitoneal tumor similar to that observed in Castleman's disease (6). In September 2015, the patient was subsequently referred to Toranomon Hospital for further evaluation and management of the mass. Considering the highly invasive nature of the surgery and the patient's refusal to undergo surgery, we decided to perform regular follow-ups semiannually using ultrasonography and MRI examination. An abdominal ultrasound carried out 5 years after the first visit to our institution revealed an increase in the size of the mass to $50 \mathrm{~mm}$, which was highly suggestive of a malignancy. Contrast-enhanced CT revealed a well-defined mass with heterogeneous contrast in the early phase and good enhancement in the delayed phase. No significant enlargement of the surrounding lymph nodes was observed. Obstruction of the splenic vein was observed. There was no invasion into other organs or the renal veins (Fig. 2). Endoscopic ultrasonography revealed a solid component in the splenic vein that appeared as a thickening of the vascular wall, and it was contiguous with the mass. Furthermore, multiple vessels were observed inside the mass. The mass formed a tumor embolus within the splenic vein, and a portion of the splenic vein had increased in size to 49x33 mm. Although the tumor growth was relatively slow, the presence of the tumor embolus suggested that the mass was a malignant tumor. MRI was not performed, as the aforementioned imaging studies were considered to be sufficient for the development of the treatment strategy preoperatively. The levels of serum tumor markers, such as carcinoembryonic antigen and carbohydrate antigen 19-9, were within the normal range. The serum amylase, lipase, elastase 1 and immunoglobulin G4 levels were within the normal limits. The differential diagnosis included pancreatic acinar cell carcinoma, pancreatoblastoma, neuroendocrine tumor, gastrointestinal stromal tumor or other types of retroperitoneal tumors. Surgical resection was decided after discussing the options with the patient.

Open radical antegrade modular pancreatectomy was performed in August 2020. No ascites, dissemination, or distant
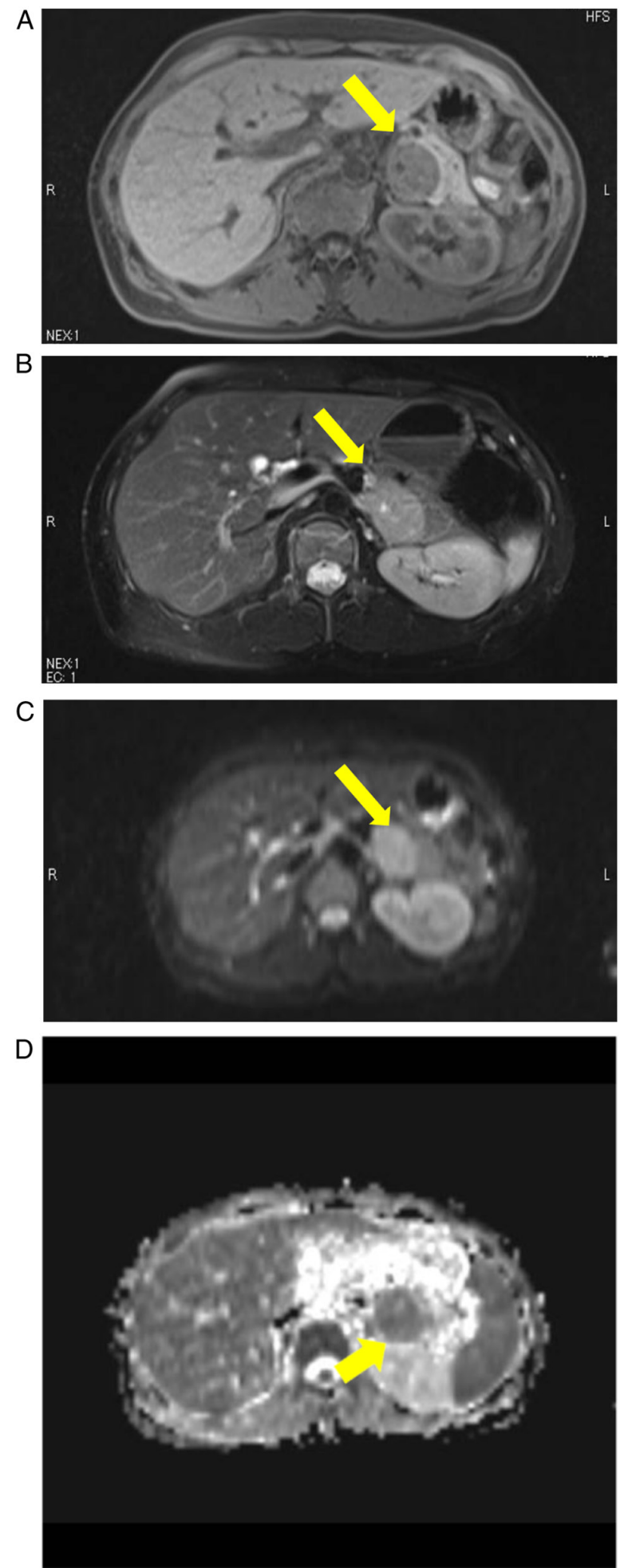

Figure 1. MRI taken at the initial hospital visit. A well-defined mass (arrows) was identified compressing the pancreas posteriorly with (A) a low signal on T1-weighted images, (B) a high signal on T2-weighted images and (C) a high signal on diffusion-weighted images. The signal intensity of the lesion on an apparent diffusion coefficient map was low. (D) The value of apparent diffusion coefficient was 0.911

metastasis were observed intraoperatively; the epiploic veins were prominently dilated. The gastrosplenic ligament was excised. The mass was a tense, elastically hard tumor located at the pancreatic tail; the pancreas appeared normal. Intraoperative ultrasound revealed a well-defined, hypervascular mass 

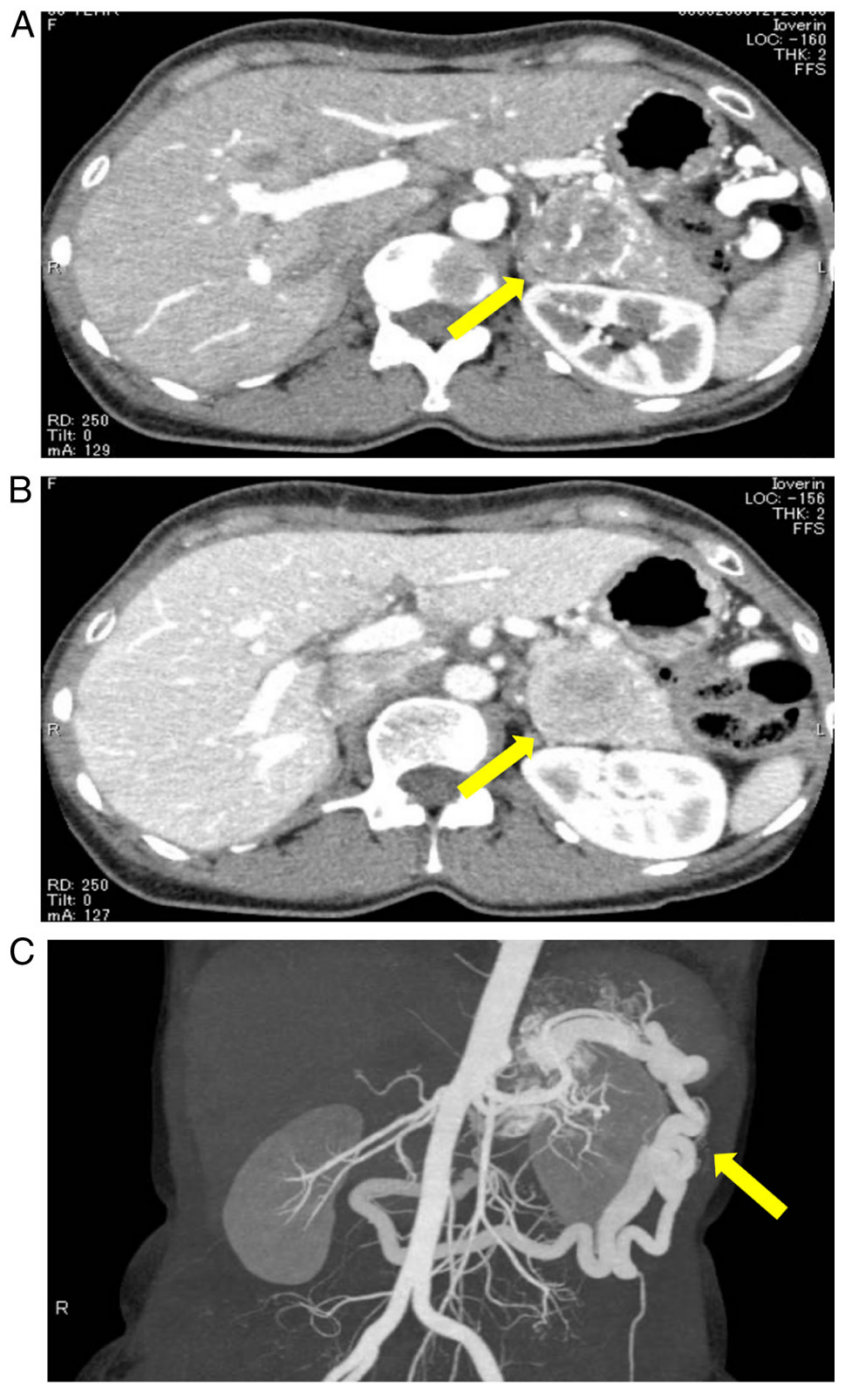

Figure 2. CT examination performed immediately prior to the surgical resection. A well-circumscribed mass (arrows) was identified, with (A) heterogenous contrast in the early phase and (B) good enhancement in the delayed phase. (C) Development of the epiploic veins (arrow) as collateral vessels was observed on angiography.

contiguous to the splenic vein. The splenic artery was ligated at its origin. The pancreas was sectioned at the level of the left border of the portal vein, and the splenic vein was subsequently divided to the terminal end. The dissection proceeded anterior to the adrenal gland in a right-to-left antegrade manner. The splenorenal ligament was divided and the spleen was mobilized. The entire specimen was then resected; $\mathrm{R} 0$ resection was successfully performed. The operative time was $106 \mathrm{~min}$, and the total intraoperative blood loss was $247 \mathrm{ml}$.

Macroscopic examination revealed that the tumor was located in the splenic vein, forming a tumor thrombus, and small tumor nodules were present in the pancreas. Histologically, the tumor was composed of spindle cells arranged in interlacing fascicles. The tumor cells were immunohistochemically positive for desmin, h-caldesmon, muscle-specific actin and $\alpha$-smooth muscle actin. Microscopic examination showed tumor infiltration beyond the wall of the splenic vein and into the surrounding soft tissue and pancreatic parenchyma. The pathological diagnosis was leiomyosarcoma arising from the splenic vein (Fig. 3).
A

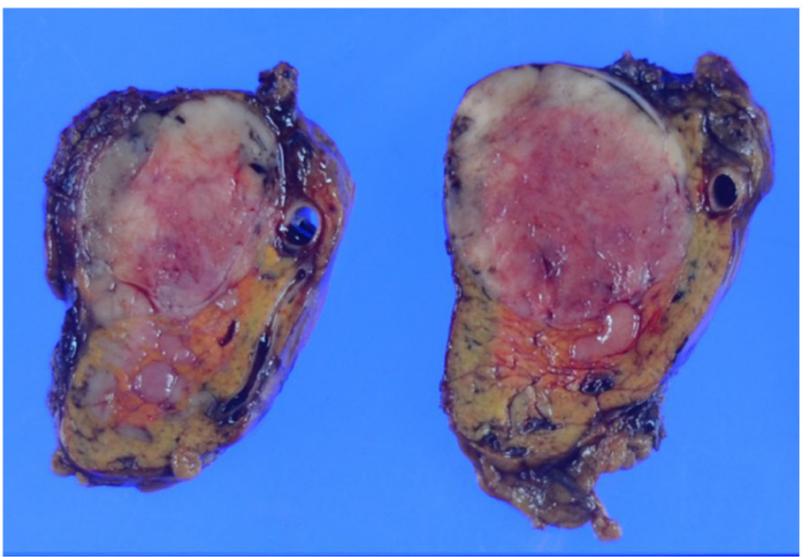

B

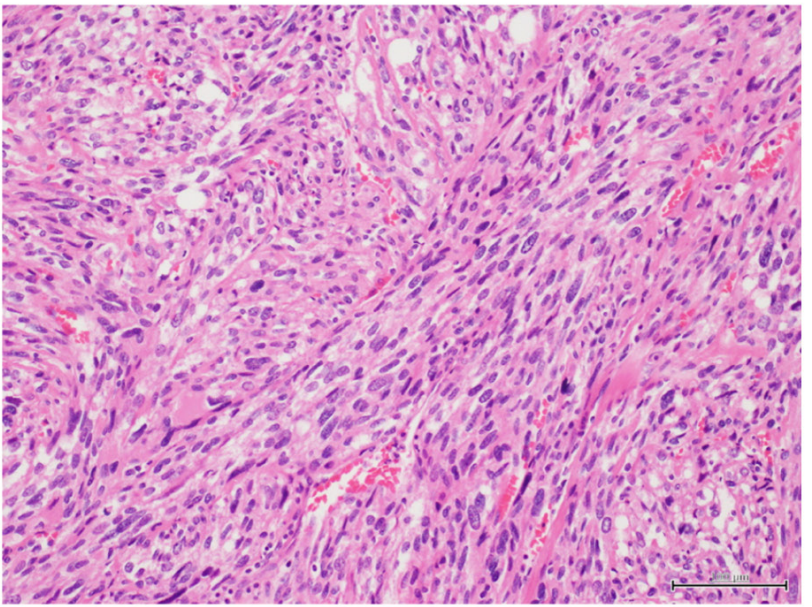

C

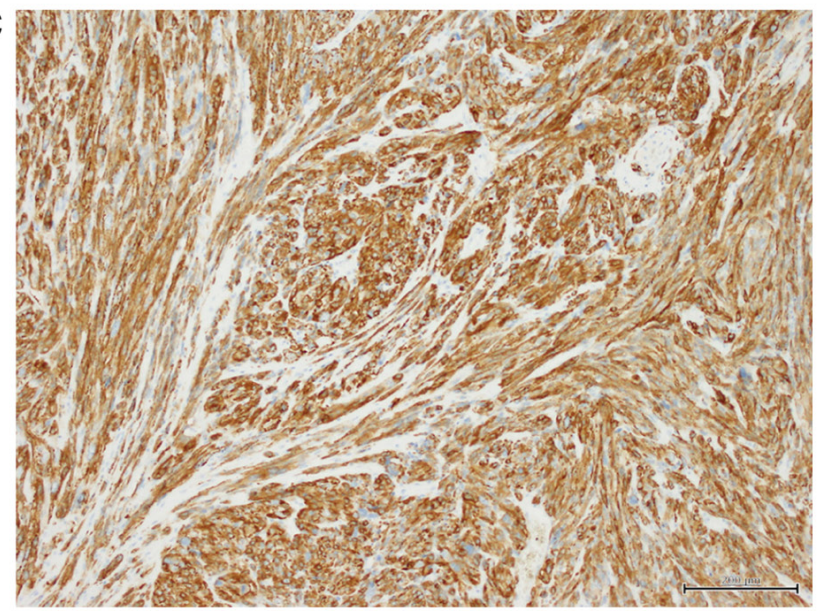

Figure 3. Surgical specimen. (A) Gross appearance. The cut surface of the tumor appeared whitish. The tumor was mostly located in the splenic vein, with small nodules in the pancreas. (B) Histological findings (H\&E staining; scale bar, $100 \mu \mathrm{m}$ ). The tumor was composed of fascicles of interlacing spindle cells with eosinophilic cytoplasm and atypical nuclei. (C) Immunostaining (scale bar, $200 \mu \mathrm{m}$ ). The tumor cells exhibited diffuse positivity for h-caldesmon.

The postoperative course was uneventful, and the patient was discharged on postoperative day 15 . Following an institutional multidisciplinary cancer board discussion, regular follow-up visits were scheduled for the patient, as no effective evidence-based chemotherapy was available in this case.

At a regular medical check-up 5 months postoperatively, abdominal ultrasound revealed four space-occupying lesions in the liver. Contrast-enhanced CT revealed four masses up 
to $15 \mathrm{~mm}$ in size, with indistinct borders, showing high and intermediate signal intensity in the early and in the portal and late phases, respectively. There were no other apparent lesions. Chemotherapy was ruled out as a treatment option, and transcatheter arterial chemoembolization (TACE) was performed using epirubicin in March 2021, since the lesions were limited to the liver. The tumors were pathologically diagnosed as leiomyosarcomas by needle biopsy.

Since the last TACE procedure, the patient has been regularly followed up with abdominal ultrasound and CT conducted at each visit; no recurrence was observed on the last follow-up visit in August 2021.

\section{Discussion}

Leiomyosarcoma is a rare malignant mesenchymal tumor originating from smooth muscle cells, which is usually located in the retroperitoneum, mesentery, omentum, uterus or subcutaneous tissue (7). Leiomyosarcomas of vascular origin account for $<2 \%$ of all cases (3). Venous leiomyosarcomas are mainly observed in women between their fifth and sixth decades of life (8). Furthermore, 75\% of large-vessel leiomyosarcomas are observed in the inferior vena cava $(9,10)$. However, those derived from the splenic vein have been reported in only a few previous studies. To the best of our knowledge, only five previous studies have reported cases of leiomyosarcoma of the splenic vein (Table I) (7,11-14).

Due to its rarity, the differential diagnosis of leiomyosarcoma of the splenic vein is challenging. However, early-stage diagnosis is crucial, as surgical resection is the only curative option in such cases $(15,16)$. Ultrasound examination is frequently performed as the first diagnostic tool. Leiomyosarcoma is characterized as a circumscribed, soft tissue mass, commonly accompanied by necrosis, cystic degeneration and hemorrhage on $\mathrm{CT}(17,18)$. In the present case, there were two possible diagnoses: A primary retroperitoneal leiomyosarcoma with secondary venous invasion or a primary venous leiomyosarcoma with secondary extravascular extension, as in the study by Niver et al (7). It was difficult to determine whether the tumor originated from the retroperitoneum or the vasculature preoperatively.

As regards the treatment strategy, en bloc resection is the only curative treatment option for this condition in the absence of disseminated disease (13), although the prognostic value of microscopic involvement of the resection margin remains disputable (16). Furthermore, in all the previous reports of leiomyosarcoma of the splenic vein, the correct diagnosis was established only after surgical resection (7,11-14). Radiation treatment is commonly performed for high-grade soft tissue sarcomas of the extremities, intermediate-grade tumors of the limbs with close margins, and recurring low-grade sarcomas (14). Among retroperitoneal sarcomas, primary leiomyosarcomas of vascular origin have the highest incidence of local recurrence and the worst long-term survival outcomes (16). The incidence of post-resection metastases is $\sim 40 \%$ in such cases $(9,19)$. The high risk of local recurrence of leiomyosarcoma may justify adjuvant radiotherapy in some cases. While chemotherapy can be performed for systemic control, the sensitivity of sarcomas to chemotherapy is considered to be low (20). Therefore, the role of chemotherapy as

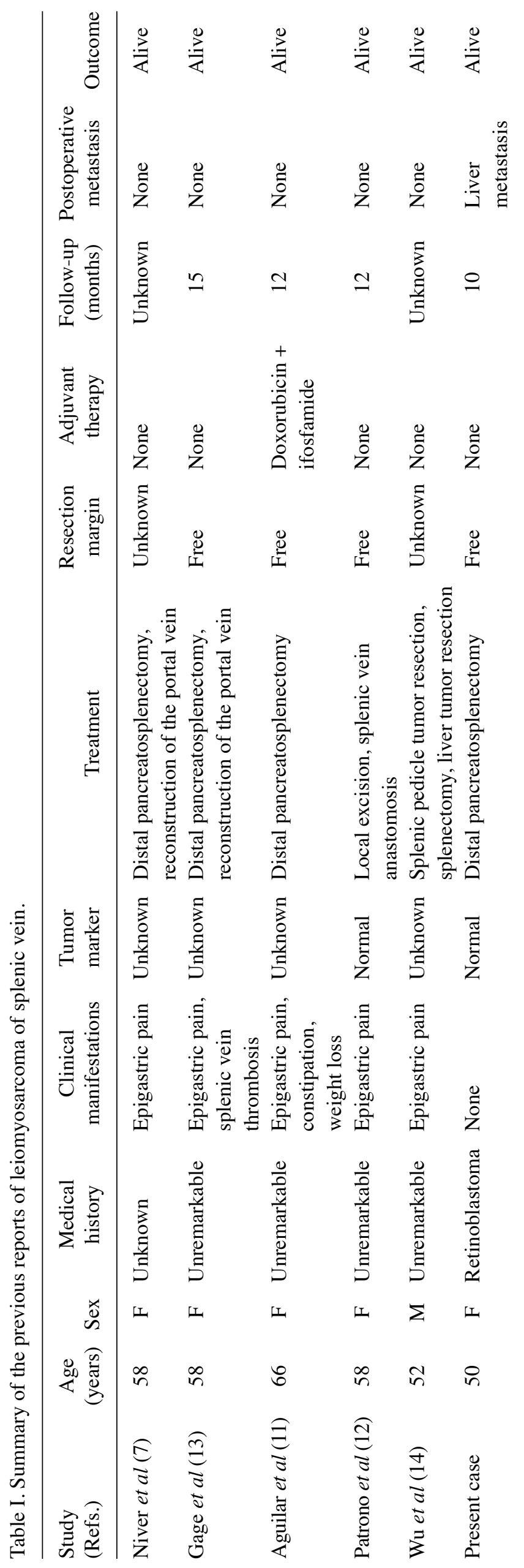


an adjuvant treatment for leiomyosarcoma for prolonging life expectancy has not yet been established (20). Evidence of prolonged survival due to adjuvant radiotherapy or chemotherapy is scarce. Among previous reports of leiomyosarcomas of the splenic vein, only one study reported that the patient underwent postoperative adjuvant therapy (Table I) (11). In a meta-analysis of soft tissue sarcomas treated with adjuvant chemotherapy, no improvement in prognosis was observed (21).

A history of hereditary retinoblastoma is associated with an increased incidence of a second, non-ocular, primary tumor (22). Retinoblastoma is an uncommon childhood tumor with an incidence of 1 per 20,000 live births (22). It can exhibit an autosomal dominant form of inheritance, although the majority of retinoblastomas are sporadic. The incidence of second non-ocular primary tumors is known to increase with time, and tumor development is associated with the loss of tumor suppressor activity caused by alterations in the Rb1 gene due to radiation, genetic mutations, or other causes (23). Although various types of secondary malignant tumors have been reported in patients with hereditary retinoblastoma, no cases of secondary tumors with splenic vein origin have previously been reported in such patients, to the best of the authors' knowledge.

In the present case, despite complete resection with a negative margin, hepatic recurrence was identified within 1 year after resection. Taking into consideration the poor outcomes of leiomyosarcomas of venous origin, successful resection may have contributed to an improved prognosis in the present case, although continuous follow-up is required.

In conclusion, the present report of a leiomyosarcoma originating in the splenic vein is, to the best of our knowledge, the sixth case of a splenic vein leiomyosarcoma reported in the English literature to date. There are currently insufficient case reports to develop clinical guidelines regarding treatment strategies for leiomyosarcoma of the splenic vein. The importance of this case lies with the rarity of leiomyosarcoma of the splenic vein and the rarity of leiomyosarcoma as a second non-ocular tumor in patients with a history of retinoblastoma. Recognition of this clinical condition at the preoperative examination stage may prove helpful for preoperative diagnosis.

\section{Acknowledgements}

Not applicable.

\section{Funding}

No funding was received.

\section{Availability of data and materials}

The datasets used and/or analyzed during the current study are available from the corresponding author on reasonable request.

\section{Authors' contributions}

MA wrote the manuscript and contributed to its design. $\mathrm{MH}$ contributed to the operation and helped draft the manuscript. DH, RK and TI performed patient follow-up and provided advice on patient treatment. YT contributed to the pathological diagnosis. MH and TI have seen and confirmed the authenticity of the raw data. All the authors have read and approved the final manuscript.

\section{Ethics approval and consent to participate}

Not applicable.

\section{Patient consent for publication}

Written informed consent was obtained from the patient regarding the publication of the case details and any associated images.

\section{Competing interests}

The authors declare that they have no competing interests.

\section{References}

1. Yang J: Primary leiomyosarcoma in the colon: A case report. Medicine (Baltimore) 97: e9923, 2018.

2. Wile AG, Evans HL and Romsdahl MM: Leiomyosarcoma of soft tissue: A clinicopathologic study. Cancer 48: 1022-1032, 1981.

3. Kevorkian J and Cento DP: Leiomyosarcoma of large arteries and veins. Surgery 73: 390-400, 1973.

4. Italiano A, Toulmonde M, Stoeckle E, Kind M, Kantor G Coindre JM and Bui B: Clinical outcome of leiomyosarcomas of vascular origin: Comparison with leiomyosarcomas of other origin. Ann Oncol 21: 1915-1921, 2010.

5. Riley DS, Barber MS, Kienle GS, Aronson JK, von Schoen-Angerer T, Tugwell P, Kiene H, Helfand M, Altman DG, Sox H, et al: CARE guidelines for case reports: Explanation and elaboration document. J Clin Epidemiol 89: 218-235, 2017.

6. Shimokihara K, Kawahara T, Kasahara R, Kasuga J, Sugiura S, Tajiri R, Uemura H and Chiba K: Retroperitoneal castleman's disease. Case Rep Oncol 12: 885-889, 2019.

7. Niver BE, Megibow AJ, Faust MJ and Rosenkrantz AB: Multidetector CT appearance of leiomyosarcoma of the splenic vein. Clin Radiol 66: 688-690, 2011.

8. Tilkorn DJ, Hauser J, Ring A, Goertz O, Stricker I, Steinau HU and Kuhnen C: Leiomyosarcoma of intravascular origin-a rare tumor entity: Clinical pathological study of twelve cases. World J Surg Oncol 8: 103, 2010.

9. Székely E, Kulka J, Miklós I and Kaliszky P: Leiomyosarcomas of great vessels. Pathol Oncol Res 6: 233-236, 2000.

10. Burke AP and Virmani R: Sarcomas of the great vessels. A clinicopathologic study. Cancer 71: 1761-1773, 1993.

11. Aguilar C, Socola F, Donet JA, Gallastegui N and Hernandez GA: Leiomyosarcoma of the splenic vein. Clin Med Insights Oncol 7: 263-268, 2013.

12. Patrono D, Molinaro L, Mazza E, Romagnoli R and Salizzoni M: Splenic vein leiomyosarcoma: Case report and review of the literature. JOP 15: 512-514, 2014.

13. Gage MJ, Newman E, Maldonado TS and Hajdu $\mathrm{CH}$ : Leiomyosarcoma of the splenic vein. J Vasc Surg 55: 1485-1487, 2012.

14. Wu W, Zhao X, Wang Y, Di C, Cai R, Zhang Y, Chen S, Zhang W and Yue X: Leiomyosarcoma of the splenic vein: A case report. Oncol Lett 14: 977-980, 2017.

15. Barbetakis N, Asteriou C, Papadopoulou FI and Stergiou E: Sarcomas of the great vessels. Is there a role for chemotherapy? Interact Cardiovasc Thorac Surg 10: 463-464, 2010.

16. Hollenbeck ST, Grobmyer SR, Kent KC and Brennan MF: Surgical treatment and outcomes of patients with primary inferior vena cava leiomyosarcoma. J Am Coll Surg 197: 575-579, 2003.

17. Hartman DS, Hayes WS, Choyke PL and Tibbetts GP: From the archives of the AFIP. Leiomyosarcoma of the retroperitoneum and inferior vena cava: Radiologic-pathologic correlation. Radiographics 12: 1203-1220, 1992. 
18. Narata M, Okuhata Y, Abe K, Takemoto A, Maebayashi T, Furuhashi S and Takahashi M: Primary leiomyosarcoma of the inferior vena cava: Case report. Abdom Imaging 35: 481-484, 2010.

19. Hilliard NJ, Heslin MJ and Castro CY: Leiomyosarcoma of the inferior vena cava: Three case reports and review of the literature. Ann Diagn Pathol 9: 259-266, 2005.

20. Clark MA, Fisher C, Judson I and Thomas JM: Soft-tissue sarcomas in adults. N Engl J Med 353: 701-711, 2005.

21. Adjuvant chemotherapy for localised resectable soft-tissue sarcoma of adults: Meta-analysis of individual data. Sarcoma meta-analysis collaboration. Lancet 350: 1647-1654, 1997.
22. Lueder GT and Smith ME: Retinoblastoma. Semin Diagn Pathol 11: 104-106, 1994.

23. Friend SH, Bernards R, Rogelj S, Weinberg RA, Rapaport JM, Albert DM and Dryja TP: A human DNA segment with properties of the gene that predisposes to retinoblastoma and osteosarcoma. Nature 323: 643-646, 1986.

c) (7) (2) This work is licensed under a Creative Commons EY NG ND Attribution-NonCommercial-NoDerivatives 4.0 International (CC BY-NC-ND 4.0) License. 